\title{
A Fully Automated Microchip Electrophoresis Analyzer for Potential Life Detection Missions
}

\author{
Maria F. Mora ${ }^{1}$, Florian Kehl ${ }^{1}$, Eric Tavares da Costa $^{1, \zeta}$, Nathan Bramall ${ }^{2}$, and Peter A. Willis ${ }^{1, *}$
}

1 Jet Propulsion Laboratory, California Institute of Technology, Pasadena, California, USA

${ }^{2}$ Leiden Measurement Technology LLC, 1230 Mountain View-Alviso Rd Suite A, Sunnyvale, CA, USA

(C) 2020 California Institute of Technology. Government sponsorship acknowledged.

Corresponding Author

*peter.a.willis@jpl.nasa.gov

Present Addresses

$\zeta$ Current address: Departamento de Química Fundamental, Instituto de Química, Universidade de São Paulo, São Paulo SP, Brazil 
Supporting Information

\section{Table of Contents}

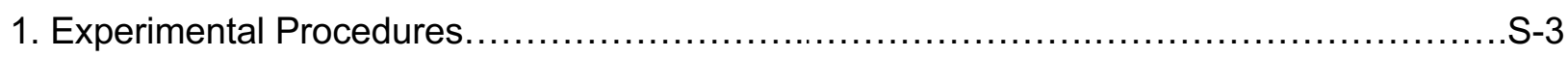

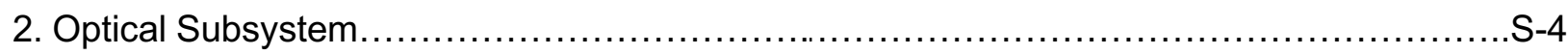

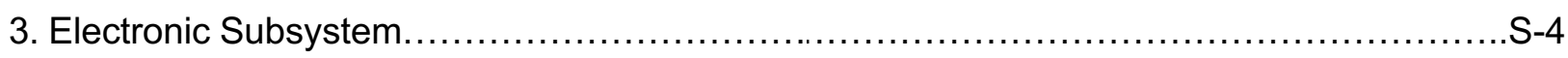

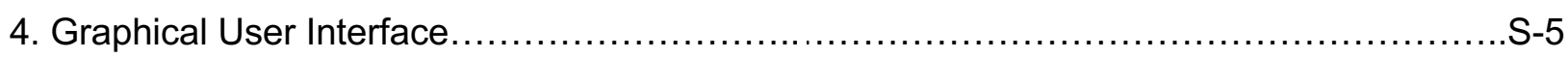

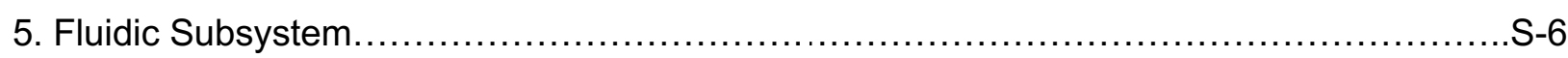

6. Amino Acid Content of Atacama Samples........................................................... 13

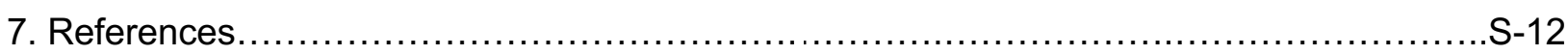




\section{Experimental Procedures}

PDMS was used to attach the Chemical Cartidge to the sample handling microchip. First, a piece of PDMS ( $3 \mathrm{~mm}$ thick) with pre-cut holes aligned with the fluidic inlets is attached to the microchip using Gel Slick ${ }^{T M}$ Solution (Lonza, Inc., Basel, Switzerland). A thin layer of uncured PDMS is later used to seal the Chemical Cartridge on top of the sample handling microchip (Figure S1).
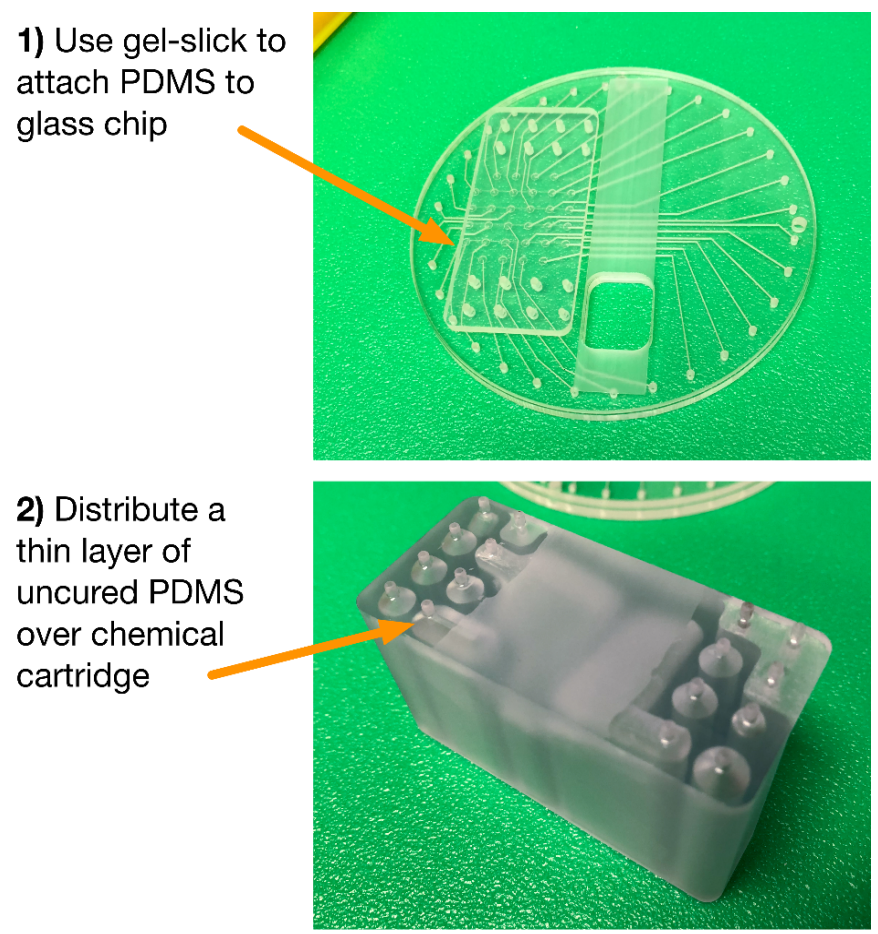

3) Place chip on top of chemical cartridge and place in the oven for two hours @ $60^{\circ} \mathrm{C}$.

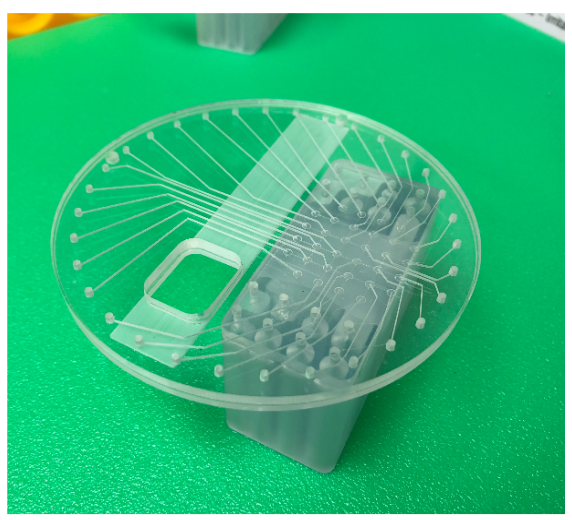

Figure S1. Steps in the assembly of the system. Thick PDMS is first attached to the glass chip using gel-slick, and then a thin layer of uncured PDMS is applied to the cartridge. It is important that this layer is the right thickness (a few $\mathrm{mm}$ ) to avoid clogging the channels by applying too much PDMS and also to avoid leakage of fluids underneath the cartridge during operation if not enough PDMS is used. 


\section{Optical Subsystem}

The LIF detection system has the standard confocal epifluorescence configuration commonly used for ME-LIF (Figure S2). Briefly, the system includes a fiber-coupled $405 \mathrm{~nm}$ laser (OBIS 405 nm LX FP $50 \mathrm{~mW}$, Coherent, Inc., Santa Clara, CA). The laser light passes through a clean-up filter (FF01-405/10, Semrock, Inc., New York, USA) and then it is reflected by a dichroic mirror (Di02-R405-15×15, Semrock, Inc., New York, USA) and focused in the channel by a custom objective lens (designed by J. Scherer ${ }^{1}$ ) from Coastal Optical Systems (a division of Jenoptik, Jena, Germany). The objective lens has a working distance of $0.81 \mathrm{~mm}$ and focuses the laser beam into a 10 to $20 \mu \mathrm{m}$ spot in the separation channel. The objective lens also has a small footprint $(2 \mathrm{~cm}$ length $\times 1 \mathrm{~cm}$ diameter), which facilitates miniaturization of the optical module. The fluorescence signal is collected by the objective lens, passed through the dichroic mirror, a long-pass filter (BLP01-405R-12.5, Semrock, Inc., New York, USA), and a notch filter (67-107, Edmund Optics, Barrington, NJ) before being focused by a collimator (F220SMA-A, $534 \mathrm{~nm}, \mathrm{f}=$ $10.92 \mathrm{~mm}$, Thorlabs, Inc., Newton, USA) into a $100 \mu \mathrm{m}$ diameter optical fiber coupled to a photoncounting head (H11890-210, Hamamatsu Photonics KK, Tokyo, Japan).

The counting head consists of a metal package photomultiplier tube along with a high-speed photon counting circuit and a high-voltage power supply unit. Both the laser and detector are integrated with their corresponding electronics. Although the laser and PMT can be controlled with their corresponding software, the manufacturers also provide libraries to use LabVIEW (National Instruments, Austin, TX, USA) or other programs to control the systems. For ease of automation, both systems are controlled with the same LabVIEW program.

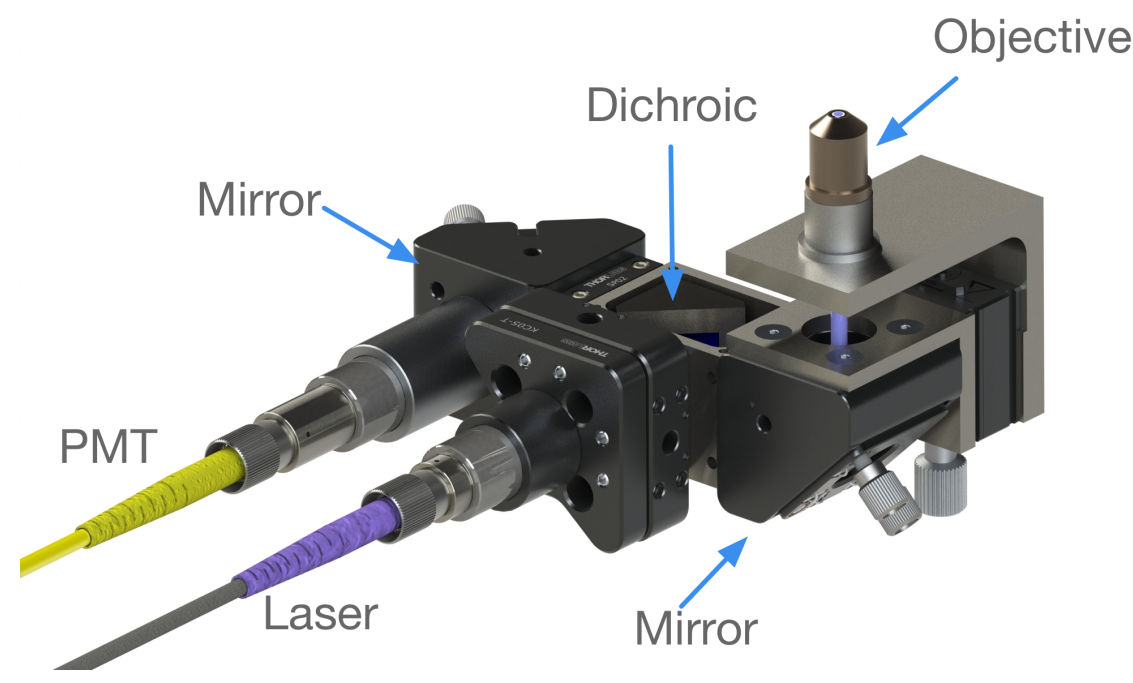

Figure S2. CAD rendering of the optical subsystem highlighting the main components.

\section{Electronic Subsystem}

"The rugged computer (DC-1100 Cincoze Rugged Intel Bay Trail Compact Fanless Computer, Logic Supply Inc., South Burlington, VT, USA) is connected to the Chemical Laptop via USB 2.0 and is used to control all operations. Inside the CL, there is a USB 2.0 hub that connects the laser, PMT, and Arduino Mega 2560 to the computer. The Arduino serves as the central control system for the $\mathrm{CL}$; it controls all of the solenoid valves, turns the pumps on/off, controls all highvoltage circuit functions, and regulates the speed of two onboard cooling fans. The Arduino Mega 
is a $5 \mathrm{~V}$ microcontroller board (Atmega2560) with 54 digital input/output pins (of which 15 can be used as pulse-width modulation (PWM) outputs) with 8 bit resolution, 16 analog inputs with a resolution of 10 bits, 4 UARTs (hardware serial ports) for communication, $1 \mathrm{SPI}$ and I2C each, and a USB connection. It contains everything needed to support the microcontroller simply by connecting to a computer with a USB cable. The firmware is written in $\mathrm{C} / \mathrm{C}++$ and provides the low-level commands to control the hardware. The Arduino Mega is directly mounted onto a custom-manufactured printed circuit board by pin headers. All of the solenoid H-bridge drivers (A3966, Allegro MicroSystems, LLC, Manchester, NH, USA) are on the same board as the highvoltage (HV) and auxiliary circuitry, with careful attention having been paid to ground planes. The HV circuitry allows for control of the voltages applied to the electrodes on the Separation Microchip, switches the HV on and off, and measures the currents flowing into and out of each branch of the ME circuit. The voltages on the $S$ and SW branches are supplied by two EMCO GP30 DC/HV DC converters (XP Power, Sunnyvale, CA, USA) and can be precisely controlled between 0 to $-3 \mathrm{kV}$. The BW voltage is generated by a SC-150N $2 \mathrm{~W}$ power supply (American High Voltage, Idaho, NV, USA) and its output voltage can be tuned from 0 to $-15 \mathrm{kV}$. The output voltage is directionally proportional to the input voltage and has a ripple of generally less than $0.8 \%$ at full power. The voltage is controlled via a low-pass filtered pulsed-width modulation signal by the microcontroller. The high voltage and return ground leads are fully isolated from the input power leads by over $1 \mathrm{~T} \Omega\left(@ 25^{\circ} \mathrm{C}\right)$. The SC-150N is reverse-input-voltage and shortcircuit protected, and the communication between the microcontroller and HV is electrically isolated by optocouplers. The buffer reservoir is grounded through a sense resistor but can be floated by opening a high-voltage relay if needed."

\section{Graphical User Interface}

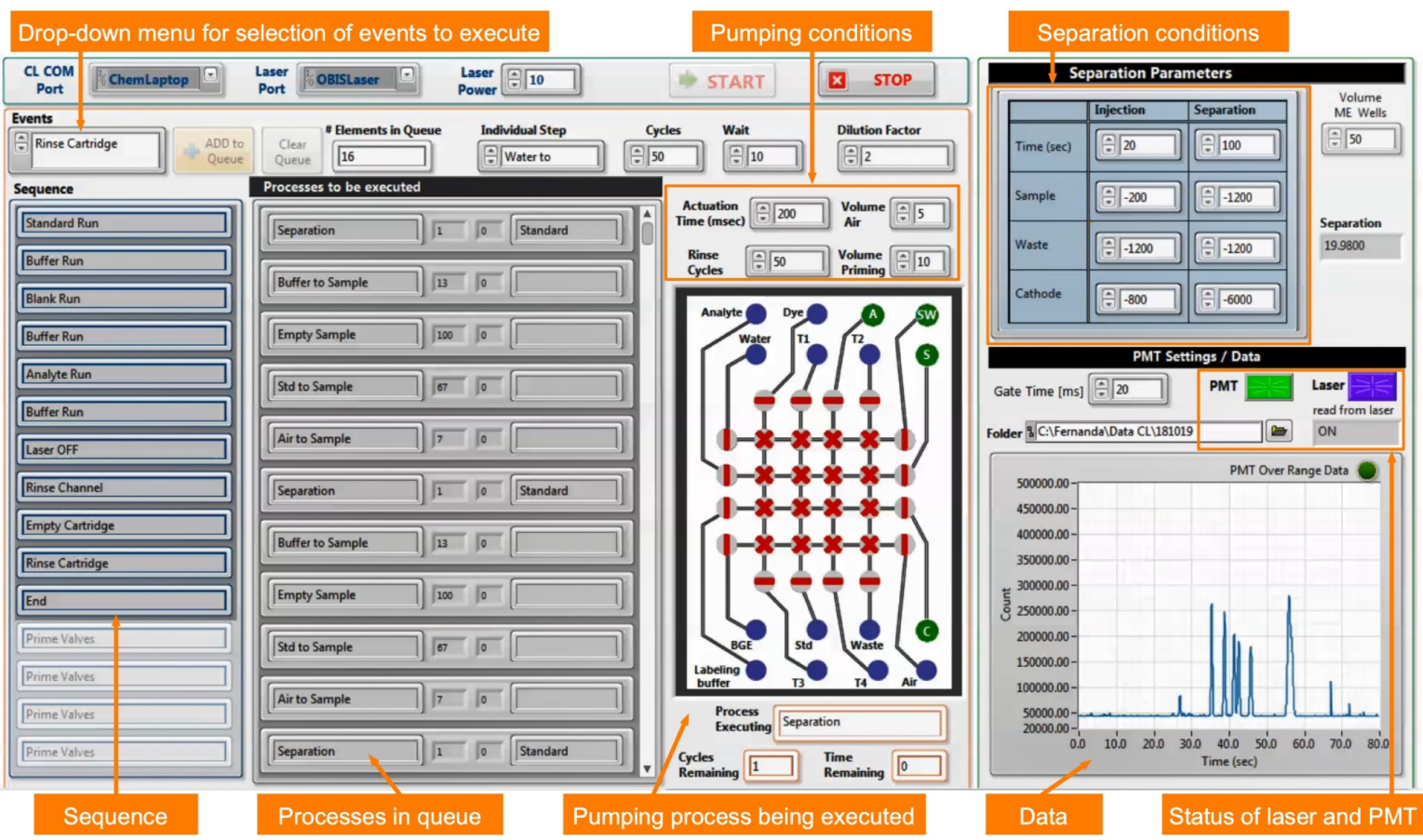


Figure S3. LabVIEW virtual instrument interface developed for automated analyses with the Chemical Laptop. The main features are highlighted. An onboard computer is used to control the CL. The program allows the user to select from a list of preloaded activities to perform automated end-to-end analyses. The preloaded programs include priming the separation microchip, sample and blank labeling, as well as rinsing of both microchips and Chemical Cartridge.

\section{Fluidic Subsystem}

\section{$\underline{\text { Overview }}$}

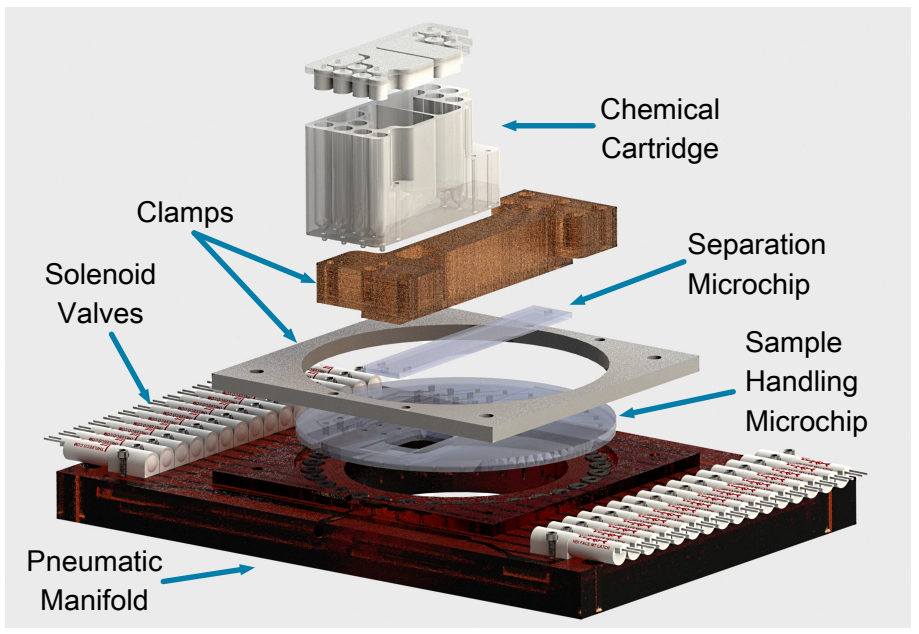

Figure S4. Exploded view rendering of the fluidic subsystem indicating the main components.

Optimization of Fluid Delivery to Microchip for Electrophoretic Analysis 


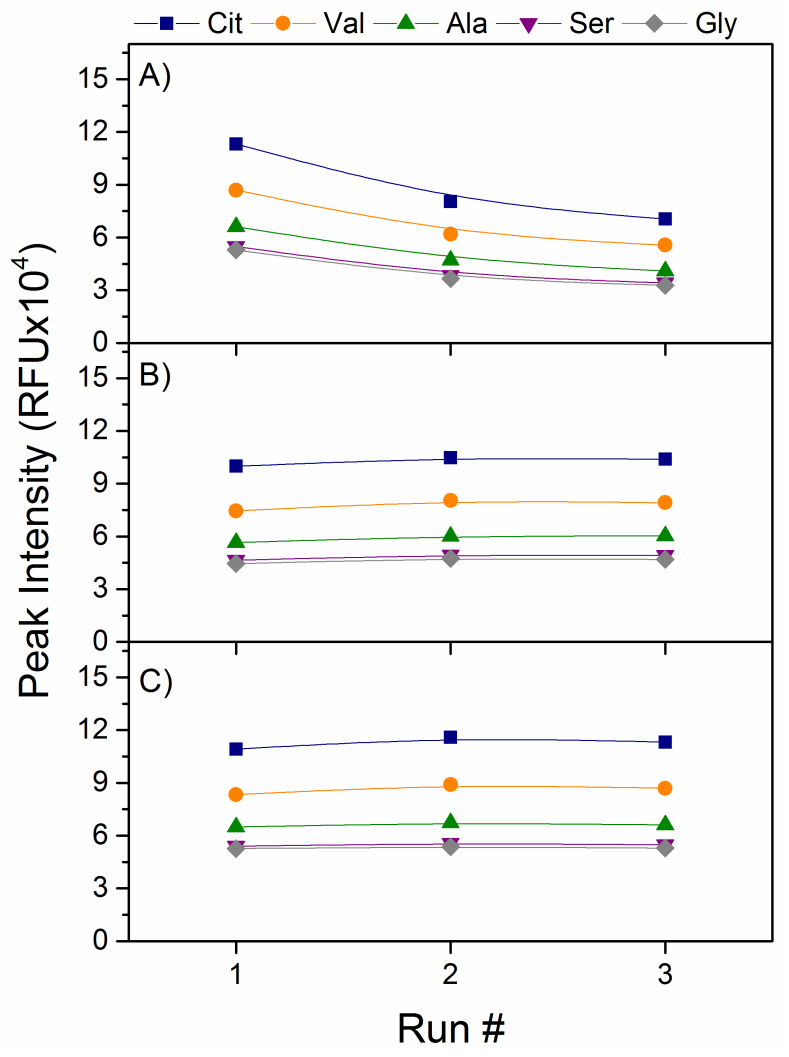

Figure S5. Fluorescent signal for three consecutive separations of a $10 \mathrm{nM}$ mixture of amino acids performed in three different situations: (A) without replacing the sample or buffer in the ME wells, B) replacing only sample for each separation, and (C) replacing sample and buffer in the ME wells for each separation. The separation conditions are $25 \mathrm{mM}$ tetraborate buffer $\mathrm{pH}$ $=9.2, \mathrm{t}_{\mathrm{INJ}}=20 \mathrm{sec}, \mathrm{V}_{\mathrm{SEP}}=6 \mathrm{kV}$.

The importance of rinsing

Figure S6 shows an example of an automated analysis where the array was not rinsed sufficiently during labeling and also between separations. The first thing to notice is that there are amino acid peaks in the blank (i.e. labeled water). In this experiment, the sequence for labeling included delivering $100 \mu \mathrm{L}$ of sample to T1, rinsing the array, and then delivering $100 \mu \mathrm{L}$ of water to T3. A preferable order for labeling operations includes first delivering water to T3 and then sample to T1, in order to avoid carry-over. The BGE runs in Figure S56 also have a residual dye peak, indicating that more rinsing is needed following analysis of both blank and sample. Based on these results, the program was modified to ensure the blank was clean, and the number of rinsing cycles was increased to reduce carry-over. 


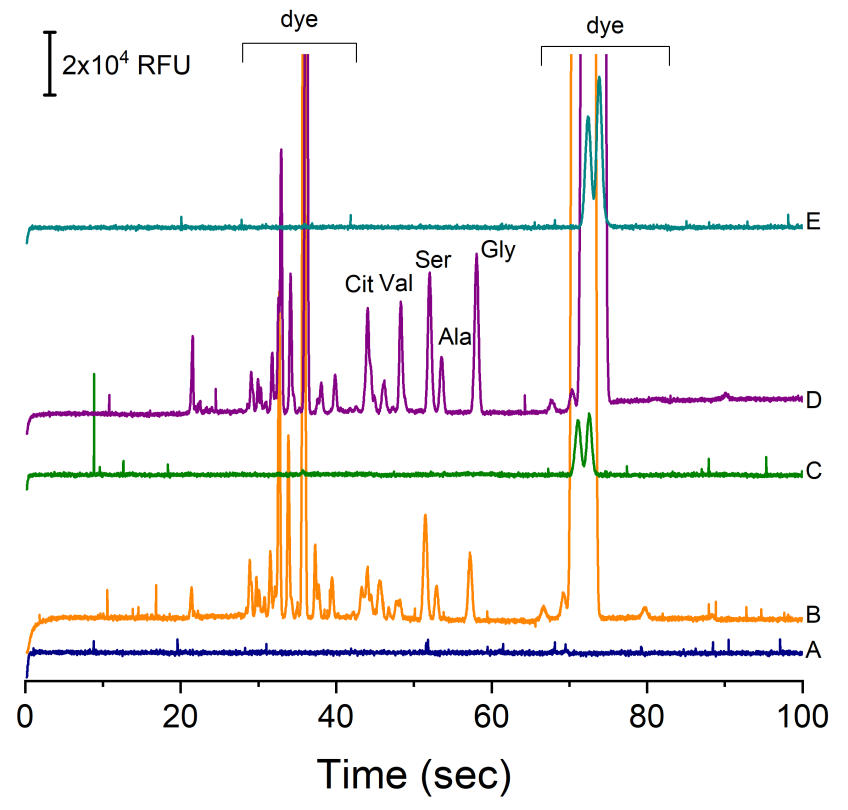

Figure S6. Results from a completely automated analysis of a sample containing $100 \mathrm{nM}$ amino acid mixture. The sample is diluted by half with buffer for labeling, resulting in a final dye concentration of $100 \mu \mathrm{M}$. Rinsing steps and repetitions are not included for clarity: A) BGE, B) blank run (labeled water), C) BGE, D) sample, and E) BGE. The separation conditions are 25 $\mathrm{mM}$ tetraborate buffer $\mathrm{pH}=9.2, \mathrm{t}_{\mathrm{INJ}}=20 \mathrm{sec}, \mathrm{V}_{\mathrm{SEP}}=6 \mathrm{kV}$

\section{Reproducibility}
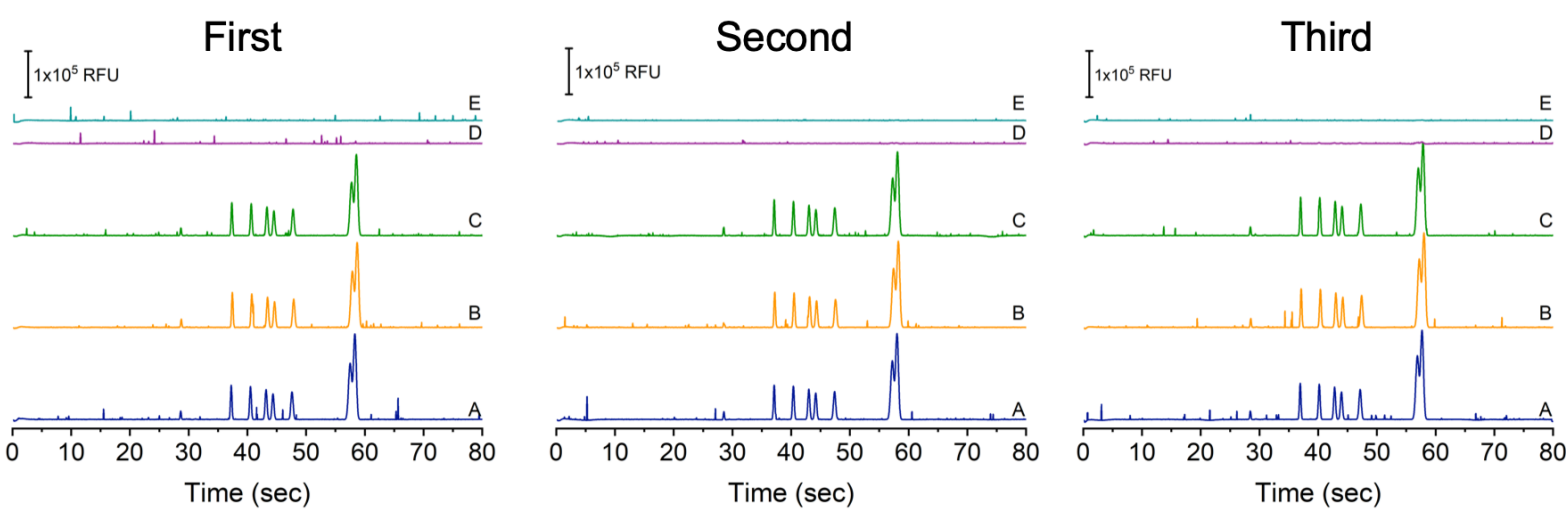

Figure S7. Electropherograms of a $10 \mathrm{nM}$ mixture of amino acids obtained with the automated program. Each analysis includes three separations of the standard mix $(A, B$, and $C)$, then the sample reservoir is rinsed with buffer twice, and two more electropherograms with buffer are obtained ( $D$ and $E$ ) before the next standard is analyzed. The figure shows three consecutive analyses. The separation conditions are $25 \mathrm{mM}$ tetraborate buffer $\mathrm{pH}=9.2, \mathrm{t}_{\mathrm{NJ}}=20 \mathrm{sec}, \mathrm{V}_{\mathrm{SEP}}$ $=6 \mathrm{kV}$. 


\section{Fluidic manipulations}

All individual pumping routines used by the automated program employ three valves for pumping. However, the total number of valves involved in each process depends on the position of the fluidic inlet and outlet in the grid. The maximum number of valves involved in a single process is nine (longest path) and the minimum is three (shortest path). In the case of pumping with the shortest path, all valves are active. However, for longer paths, there are always some valves that are not active during pumping. They are opened at the beginning of the process and they stay open for however many number of cycles are performed. We call these access valves. The valves that connect to the reservoir where the liquid comes from are called Access Valves In, and the ones connected to the reservoir that receives the liquid are called Access Valves Out. The assignment of valves for each pumping process obeys the following rules:

1. Always use the minimum number of valves for a particular process.

2. If the number of total access valves is even, the same number of valves is assigned for In and Out.

3. If the number of total access valves is odd, the extra valve is assigned as In.

4. When possible, use the valves that align with one of the array rinsing paths in order to minimize the time required for rinsing steps. The four rinsing processes for the array are shown in Figure S8. Figure S9 shows some examples of pumping processes which utilize differing numbers of access valves.

Based on these rules, all pumping processes needed for automation were determined and incorporated into the software. The valve numbers used for each pumping process are detailed in Table S1. 

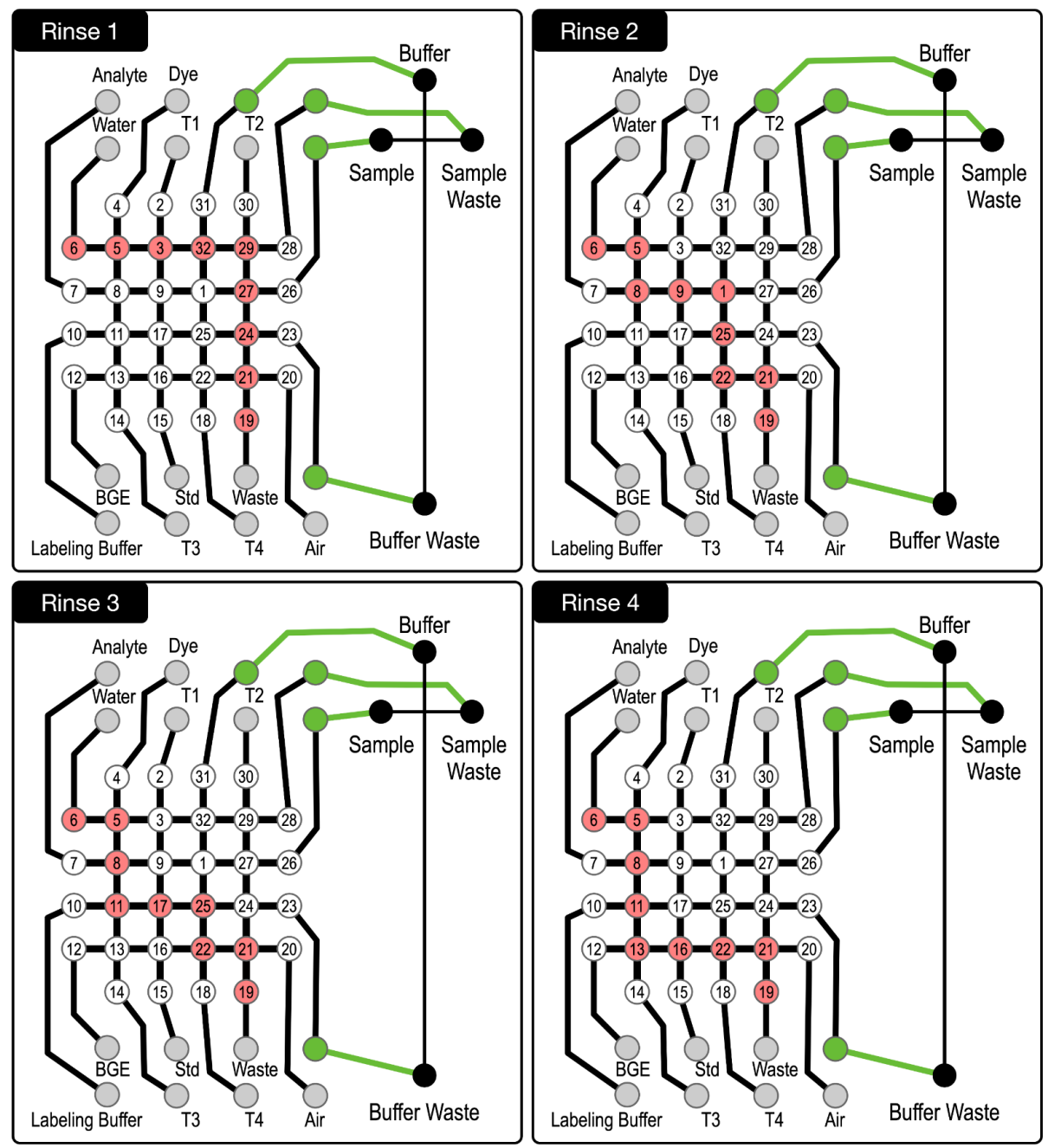

Figure S8. Pumping processes used to clean the $4 \times 4$ array. 


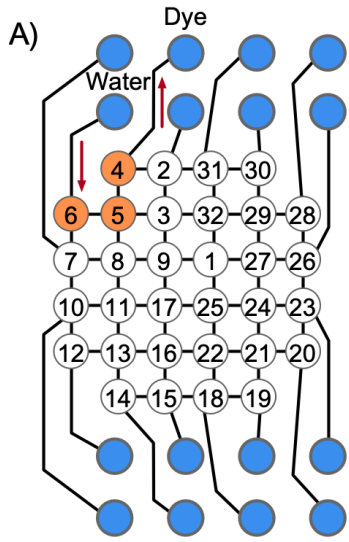

B)

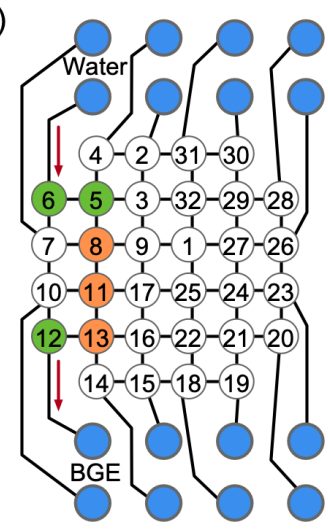

C)

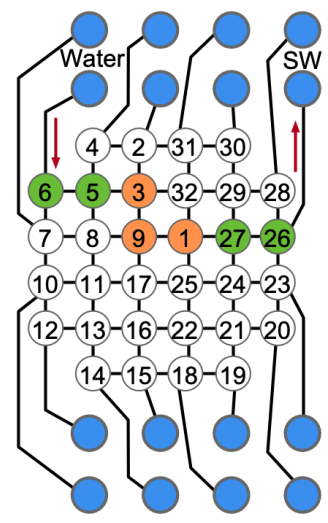

D)

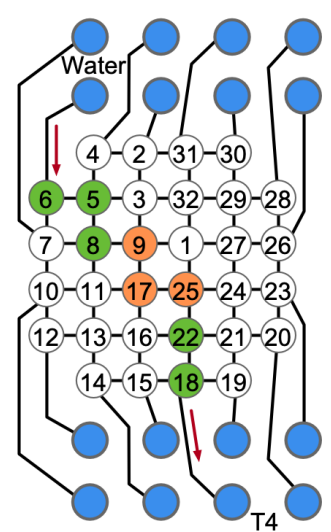

Figure S9. Examples of pumping routines using different numbers of access valves: A) Water to Dye, B) Water to BGE, C) Water to Sample Waste, and D) Water to T4. Access valves are green while valves involved with active pumping are orange. Arrows indicate the direction of fluid movement.

Table S1. Valves used for each fluidic handling process.

\begin{tabular}{|l|c|c|c|c|c|c|c|c|c|}
\hline \multicolumn{1}{|c|}{ Process } & \multicolumn{3}{c|}{$\begin{array}{c}\text { Input Access } \\
\text { Valves }\end{array}$} & \multicolumn{3}{c|}{ Pumping Valves } & \multicolumn{3}{c|}{$\begin{array}{c}\text { Output Access } \\
\text { Valves }\end{array}$} \\
\hline Rinse 1 & 6 & 5 & 3 & 32 & 29 & 27 & 24 & 21 & 19 \\
\hline Rinse 2 & 6 & 5 & 8 & 9 & 1 & 25 & 22 & 21 & 19 \\
\hline Rinse 3 & 6 & 5 & 8 & 11 & 17 & 25 & 22 & 21 & 19 \\
\hline Rinse 4 & 6 & 5 & 8 & 11 & 13 & 16 & 22 & 21 & 19 \\
\hline Water to Analyte & 6 & & & 5 & 8 & 7 & & & \\
\hline Water to BGE & 6 & 5 & & 8 & 11 & 13 & 12 & & \\
\hline Water to Dye & & & & 6 & 5 & 4 & & & \\
\hline Water to Labeling Buffer & 6 & & & 5 & 8 & 11 & 10 & & \\
\hline Water to Std & 6 & 5 & & 8 & 9 & 17 & 16 & 15 & \\
\hline Water to T1 & 6 & & & 5 & 3 & 2 & & & \\
\hline Water to T2 & 6 & 5 & & 3 & 32 & 29 & 30 & & \\
\hline Water to T3 & 6 & 5 & & 8 & 11 & 13 & 14 & & \\
\hline Water to T4 & 6 & 5 & 8 & 9 & 17 & 25 & 22 & 18 & \\
\hline & & & & & & & & & \\
\hline Water to Buffer & 6 & & & 5 & 3 & 32 & 31 & & \\
\hline Water to Sample Waste & 6 & 5 & & 3 & 32 & 29 & 28 & & \\
\hline Water to Sample & 6 & 5 & & 3 & 9 & 1 & 27 & 26 & \\
\hline Water to Buffer Waste & 6 & 5 & 8 & 9 & 17 & 25 & 24 & 23 & \\
\hline Air to Buffer & 20 & 21 & & 24 & 25 & 1 & 32 & 31 & \\
\hline Air to Sample Waste & 20 & 21 & & 24 & 27 & 29 & 28 & & \\
\hline Air to Sample & 20 & & & 21 & 24 & 27 & 26 & & \\
\hline Air to Buffer Waste & 20 & & & 21 & 24 & 23 & & & \\
\hline Analyte to waste & 7 & 8 & 9 & 17 & 25 & 24 & 21 & 19 & \\
\hline BGE to waste & 12 & 13 & & 16 & 22 & 21 & 19 & & \\
\hline Dye to waste & 4 & 5 & 8 & 9 & 1 & 25 & 24 & 21 & 19 \\
\hline Labeling buffer to waste & 10 & 11 & & 17 & 25 & 24 & 21 & 19 & \\
\hline
\end{tabular}


Supporting Information

\begin{tabular}{|c|c|c|c|c|c|c|c|c|c|}
\hline Std to waste & 15 & & & 16 & 22 & 21 & 19 & & \\
\hline BGE to Buffer & 12 & 13 & 11 & 17 & 9 & 1 & 32 & 31 & \\
\hline BGE to Sample Waste & 12 & 13 & 11 & 17 & 9 & 1 & 27 & 29 & 28 \\
\hline BGE to Sample & 12 & 13 & 11 & 17 & 9 & 1 & 27 & 26 & \\
\hline BGE to Buffer Waste & 12 & 13 & & 16 & 17 & 25 & 24 & 23 & \\
\hline Empty Buffer & 31 & 32 & & 1 & 25 & 24 & 21 & 19 & \\
\hline Empty Sample Waste & 28 & 29 & & 27 & 24 & 21 & 19 & & \\
\hline Empty Sample & 26 & & & 27 & 24 & 21 & 19 & & \\
\hline Empty Buffer Waste & 23 & & & 24 & 21 & 19 & & & \\
\hline Std to Sample Well & 15 & 16 & & 17 & 9 & 1 & 27 & 26 & \\
\hline Analyte to $\mathrm{T} 1$ & 7 & & & 8 & 0 & 3 & 2 & & \\
\hline Dye to T1 & 4 & & & 5 & 3 & 2 & & & \\
\hline BGE to T1 & 12 & 13 & & 11 & 17 & 9 & 3 & 2 & \\
\hline Labeling Buffer to T1 & 10 & 11 & & 17 & 9 & 3 & 2 & & \\
\hline T1 to T2 & 2 & & & 3 & 32 & 29 & 30 & & \\
\hline T1 to T3 & 2 & 3 & & 9 & 17 & 11 & 13 & 14 & \\
\hline T1 to T4 & 2 & 3 & & 9 & 17 & 25 & 22 & 18 & \\
\hline T1 to Sample Well & 2 & 3 & & 9 & 1 & 27 & 26 & & \\
\hline T1 to Waste & 2 & 3 & 9 & 17 & 25 & 24 & 21 & 19 & \\
\hline Analyte to T2 & 7 & 8 & & 9 & 1 & 27 & 29 & 30 & \\
\hline Dye to T2 & 4 & 5 & & 3 & 32 & 29 & 30 & & \\
\hline BGE to T2 & 12 & 13 & 11 & 17 & 25 & 1 & 27 & 29 & 30 \\
\hline Labeling Buffer to T2 & 10 & 11 & 17 & 25 & 24 & 27 & 29 & 30 & \\
\hline T2 to T1 & 30 & & & 29 & 32 & 3 & 2 & & \\
\hline T2 to T3 & 30 & 29 & 27 & 1 & 9 & 17 & 16 & 13 & 14 \\
\hline T2 to T4 & 30 & 29 & & 32 & 1 & 25 & 22 & 18 & \\
\hline T2 to Sample Well & 30 & & & 29 & 27 & 26 & & & \\
\hline T2 to Waste & 30 & 29 & & 27 & 24 & 21 & 19 & & \\
\hline Analyte to T3 & 7 & & & 8 & 11 & 13 & 14 & & \\
\hline Dye to T3 & 4 & 5 & & 8 & 11 & 13 & 14 & & \\
\hline BGE to T3 & & & & 12 & 13 & 14 & & & \\
\hline Labeling Buffer to T3 & 10 & & & 11 & 13 & 14 & & & \\
\hline T3 to T1 & 14 & 13 & & 11 & 17 & 9 & 3 & 2 & \\
\hline T3 to T2 & 14 & 13 & 11 & 17 & 25 & 1 & 27 & 29 & 30 \\
\hline T3 to T4 & 14 & & & 13 & 16 & 22 & 18 & & \\
\hline T3 to Sample Well & 14 & 13 & 16 & 17 & 25 & 1 & 27 & 26 & \\
\hline T3 to Waste & 14 & 13 & & 16 & 22 & 21 & 19 & & \\
\hline Analyte to T4 & 7 & 8 & & 9 & 17 & 25 & 22 & 18 & \\
\hline Dye to T4 & 4 & 5 & 3 & 9 & 17 & 25 & 22 & 18 & \\
\hline BGE to T4 & 12 & & & 13 & 16 & 22 & 18 & & \\
\hline Labeling Buffer to T4 & 10 & 11 & & 17 & 25 & 22 & 18 & & \\
\hline T4 to T1 & 18 & 22 & & 25 & 1 & 9 & 3 & 2 & \\
\hline T4 to T2 & 18 & 22 & & 25 & 1 & 32 & 29 & 30 & \\
\hline T4 to T3 & 18 & & & 22 & 16 & 13 & 14 & & \\
\hline T4 to Sample Well & 18 & 22 & & 25 & 1 & 27 & 26 & & \\
\hline T4 to Waste & 18 & & & 22 & 21 & 19 & & & \\
\hline
\end{tabular}




\section{Amino Acid Content of Atacama Samples}

Table S2. Concentration of amino acids detected in Atacama soil samples during the field campaign.

\begin{tabular}{|c|c|c|c|c|c|c|c|}
\hline \multirow[b]{2}{*}{$\begin{array}{l}\text { Amino } \\
\text { Acid }\end{array}$} & \multicolumn{2}{|c|}{ Sample 1} & \multicolumn{2}{|c|}{ Sample 2} & \multicolumn{2}{|c|}{ Sample 3} & Sample 4 \\
\hline & $\begin{array}{c}\text { Extract } \\
(\mathrm{nM})\end{array}$ & $\begin{array}{c}\text { Soil }{ }^{*}(\mathrm{ng} / \mathrm{g} \\
\text { or } \mathrm{ppb})\end{array}$ & $\begin{array}{c}\text { Extract } \\
(\mathrm{nM})\end{array}$ & $\begin{array}{l}\text { Soil }{ }^{*}(n g / g \\
\text { or } p p b)\end{array}$ & $\begin{array}{c}\text { Extract } \\
(\mathrm{nM})\end{array}$ & $\begin{array}{c}\text { Soil }{ }^{*}(\mathrm{ng} / \mathrm{g} \\
\text { or } \mathrm{ppb})\end{array}$ & $\begin{array}{c}\text { Extract } \\
(\mathrm{nM})\end{array}$ \\
\hline Leucine & LOD & - & LOD & - & $7.8 \pm 0.4$ & $4.1 \pm 0.2$ & $N^{a}$ \\
\hline Valine & LOD & - & $2.9 \pm 0.5$ & $1.3 \pm 0.3$ & $5.9 \pm 0.2$ & $2.8 \pm 0.1$ & $\mathrm{ND}^{\mathrm{a}}$ \\
\hline Serine & $18 \pm 2$ & $7.6 \pm 0.7$ & $33 \pm 8$ & $14 \pm 3$ & $44 \pm 3$ & $18 \pm 1$ & $\mathrm{ND}^{\mathrm{a}}$ \\
\hline Alanine & $49 \pm 4$ & $17 \pm 1$ & $11 \pm 2$ & $4.1 \pm 0.6$ & $6.4 \pm 0.4$ & $2.3 \pm 0.2$ & $\mathrm{ND}^{\mathrm{a}}$ \\
\hline Glycine & $26 \pm 2$ & $7.8 \pm 0.6$ & $22 \pm 4$ & $7 \pm 1$ & $22 \pm 1$ & $6.4 \pm 0.4$ & $\mathrm{ND}^{\mathrm{a}}$ \\
\hline
\end{tabular}

*Assumes $0.5 \mathrm{~g}$ of soil was delivered to the extractor sample cup by the robotic sample handling system. This is the nominal sample volume, but there was no operational verification of this value in the field. $2 \mathrm{~mL}$ total volume of water was used for subcritical water extraction.

ND: not detected above the detection limit, LOD: amino acids present at the limit of detection.

\section{References}

(1) Skelley, A. M.; Scherer, J. R.; Aubrey, A. D.; Grover, W. H.; Ivester, R. H. C.; Ehrenfreund, P.; Grunthaner, F. J.; Bada, J. L.; Mathies, R. A., Proc. Nat. Acad. Sci. 2005, 102 (4), 1041-1046. 